\title{
Coordinated control of H Bridge Power Conversion System for Photovoltaic integration
}

\author{
Sun Jian ${ }^{1,}$, Wang Qiang ${ }^{2, b}$ \\ ${ }^{1}$ College of Electrical Engineering \& New Energy, China Three Gorges University, Yichang, China \\ ${ }^{2}$ College of Electrical Engineering \& New Energy, China Three Gorges University, Yichang, China \\ asunjian2009@ctgu.edu.cn, bwq00518@sohu.com
}

\begin{abstract}
Keywords: Photovoltaic(PV) integration, $H$ bridge power conversion system(HB-PCS), Coordinated control, change rate control.

Abstract. According to the requirement of grid connected photovoltaic power station and analysis of the topology structure and features of $\mathrm{H}$ bridge power conversion system(HB-PCS), a coordinated control method was presented in this paper. The method set current reference of HB-PCS to compensate reactive and active power with consideration of PV station specification of active power smoothing and voltage stability. Meanwhile, the battery in charging and discharging was protected by using fully rapid response of change rate control. Simulation results show that HB-PCS with the coordinated control can effectively promote the performance of PV integration, and enhance low voltage ride through capability, and provide a certain dynamic reactive power support for power grid.
\end{abstract}

\section{Introduction}

Large capacity MW photovoltaic power generation system, also known as photovoltaic power plant, usually requires grid-connected operation. However, PV output power fluctuates greatly due to natural conditions such as sunshine and temperature, so PV source isn't constant voltage one as well as constant current one. PV in grid-connected operation will cause a great impact on power grid, and it is hard to participate scheduling for PV source[1].

When PV power is highly permeable or output increases, the faults from grid will cause PV station trip. Because PV reconnection with grid needs times after fault recovery, power shortage during this time will make adjacent PV stations trip, even lead to blackout, then safety and stability of grid operation will decrease[2]. Therefore, research on LVRT technology about large PV station should be carried out to ensure the safe and stable operation of the grid-connected PV station.

Utilization of energy storage to stabilize random output power fluctuation of renewable energy is one of current research focuses. In PV station case, more experts have recognized the importance of stabilizing power fluctuation[3]. Combination energy storage into PV station was presented to realize PV station flexible connection with grid in [4]. Compound energy storage stabilization ways based battery storage and super capacitor demonstrated that storage system could provide transient power to maintain system reliable operation, but its control is too complicated and high cost[5].

In this work, a coordinated control method for active and reactive power is studied in the case of SOC equilibrium of the battery pack.

\section{Structure of HB-PCS}

The topology structure of HB-PCS based battery is shown in Fig.1. HB-PCS is composed of high frequency isolation half bridge DC/DC converter and cascade $\mathrm{H}$ bridge DC/AC converter in Fig. 1. All HB-PCS modules have same structure and control method, which is easy to be modularized and to set redundant units. The structure can execute high voltage bidirectional large power conversion, and battery packs are in better adaptive status. It is also to increase output voltage levels, and to outlet sinusoidal waveforms with good harmonic characteristics. Because of the above advantages, HB-PCS is very suitable for application in the new energy power generation, especially when it is combined with batteries. 


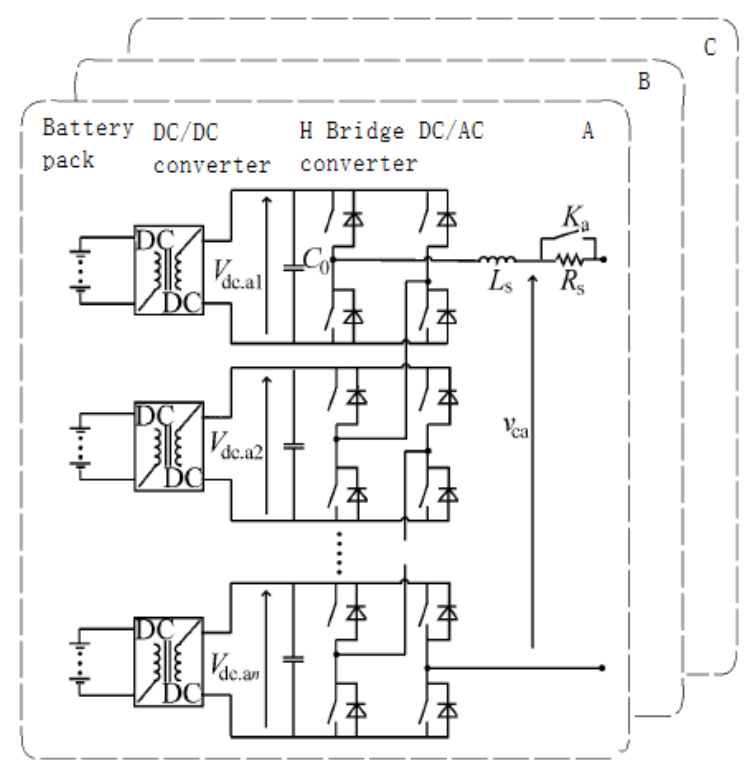

Fig.1 Structure of HB-PCS based battery

\section{Coordinated control method for HB-PCS}

Fundamentals of coordinated control method. Coordinated control method for HB-PCS presented in this work is given in Fig.2, where $P_{p v}^{*}$ is reference output value for active power of PV station after compensation, and $P_{p v}$ is actual output value for active power, and $P_{B}^{*}$ and $P_{B}$ are reference output value and actual value for active power of battery storage system respectively, and $V_{P C C}^{*}$ and $V_{P C C}$ are reference and actual voltage value at injection site. $\operatorname{SOC}_{k}(k=1,2, \mathrm{~L}, n)$ are $\mathrm{SOC}$ values for cascade batteries.

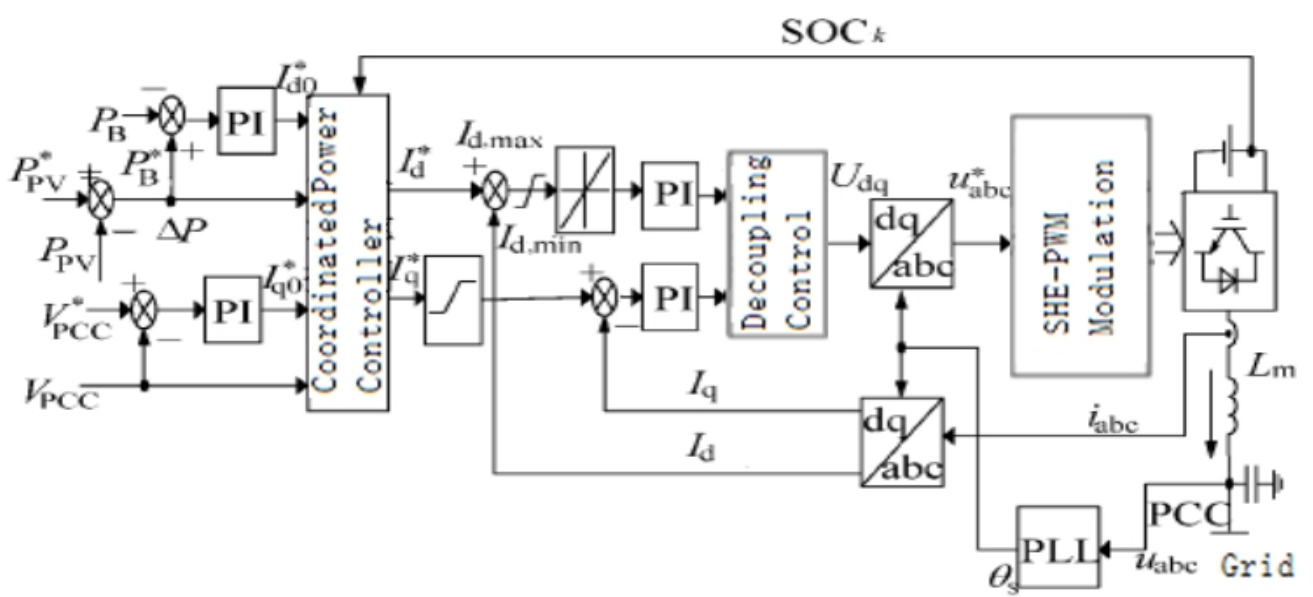

Fig. 2 Coordinated control method of HB-PCS based battery pack

The control system has dual closed loops. The inner one is current loop, which amplitude and change rate are limited in a range to protect them. The outer one is active power and voltage loop, to which change rate control and coordinated control are introduced. Coordinated power control decides reference current value of active and reactive power for compensator depending on $P_{p v}$ and $V_{P C C}$, with consideration of the requirement of change rate. Under the conditions of SOC equilibrium of battery pack, power four quadrant adjustment capability of device can be made full use to smooth output power of PV station and let PCC voltage stable.

Change rate control. Change rate is a characteristic variable with consensus between new energy power ventures and independent power manufactures[9]. In joint operation of PV and energy storage, output power change rate of battery device must track sudden change of PV station to ensure power 
from storage system to compensate PV power fluctuation. The change rate only refers to that of active power, and reactive power coupled can be modified independently.

Active and reactive power compensation. If active power output of PV station is completely smooth, the energy storage system capacity will be very large[9]. However, only to reduce large output power fluctuation and improve grid stability, the required energy storage capacity is small with more practical application. Its schematic diagram is shown in Fig.3.

It is regulated in Ref. [1] that large and middle scale PV stations should have some tolerability of abnormal voltage to ride through the grid abnormal voltage to reduce loss of the grid. When the voltage at the point of grid-connected runs in the regulated range, PV station must keep in grid-connected without interrupt. If it overruns that range, PV station is allowed to stop transmitting power to gird. Its schematic diagram is shown in Fig.4.

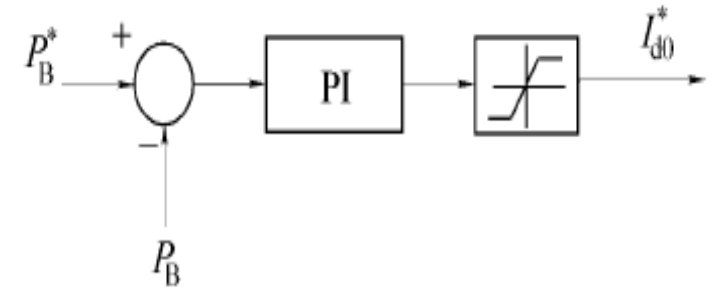

Fig.3 Diagram of active power compensation

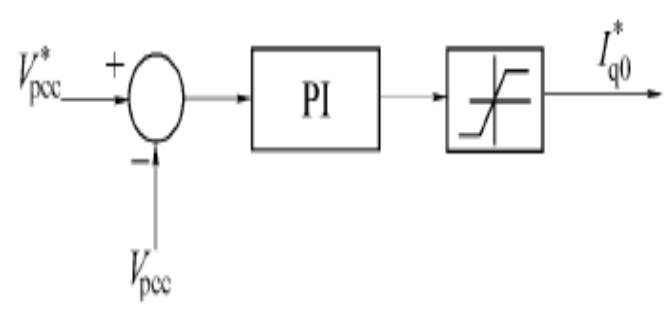

Fig.4 Diagram of active power compensation

Cooperative logic control for complex power. Coordinated power control needs to smooth active power output of PV station, and keep voltage at common point stable and cooperate and optimize charging and discharging of battery pack. Therefore, the fundamentals of coordinated control:

1) Judge whether battery needs balancing adjustment or not based on its SOC status, and algorithm in details refers to Ref. [11].

2) Calculate target current initial values $I_{d 0}^{*}$ and $I_{q 0}^{*}$ according to compound power compensation approach.

3) Set critical value $\Delta P_{m}$ of actual output active power and smoothing target error, and determine if large fluctuation from PV output power is compensated or not to protect battery pack from regulating and charging or discharging frequently.

4 ) Due to limits of power of current convertor, when $\sqrt{I_{d 0}^{* 2}+I_{q 0}^{* 2}}>I_{\max }$, active power prior control or reactive power prior control is adopted depending on voltage value at PCC point.

\section{Case Study}

Simulation models for HB-PCS and PV station integration are built with PSCAD and Matlab/Simulink in Fig.1, where installment capability of PV station is $10 \mathrm{MW}$, and rated power for storage system is $\pm 1 \mathrm{MW}$, connected with $35 \mathrm{kV}$ grid through transformer.

Some tests on low voltage ride through ability are executed. Battery storage system can stabilize the output power fluctuation of PV station. Fault occurs at $10 \mathrm{kV}$ bus bar in around $0.3 \mathrm{~s}$, which results in voltage at PCC point down to 0.7 p.u. After constant voltage reactive power compensation, it can be rise to 0.9 p.u. The fault is removed after $0.08 \mathrm{~s}, \mathrm{AC}$ bus voltage recover quickly. Simulation results are shown in Fig. 5. While storage compensation equipment fails, voltage at PCC point maintains stable, which meets LVRT requirements.

Due to limited space, sudden bus bar voltage rising fault simulation curves are ignored. From the above cases study, when storage device is in pure reactive power adjustment mode, its current is almost zero, namely, during PV station without output in night, it runs in STATCOM mode to provide reactive power support to grid. 


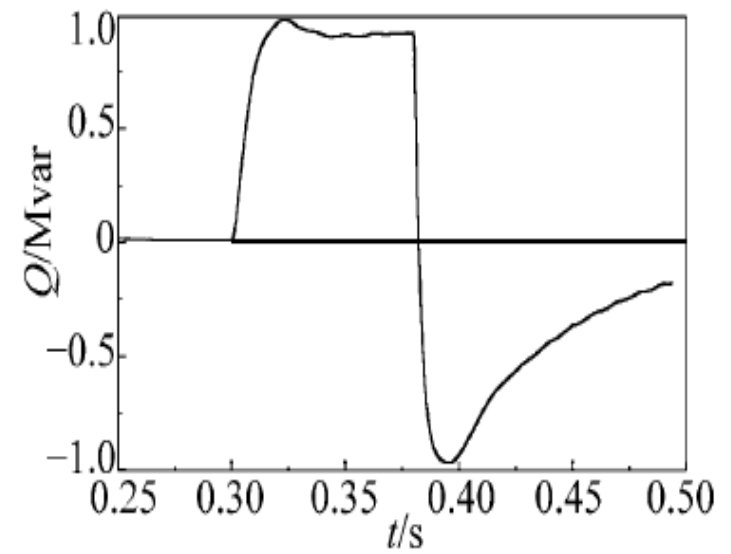

(a) Reactive power compensation

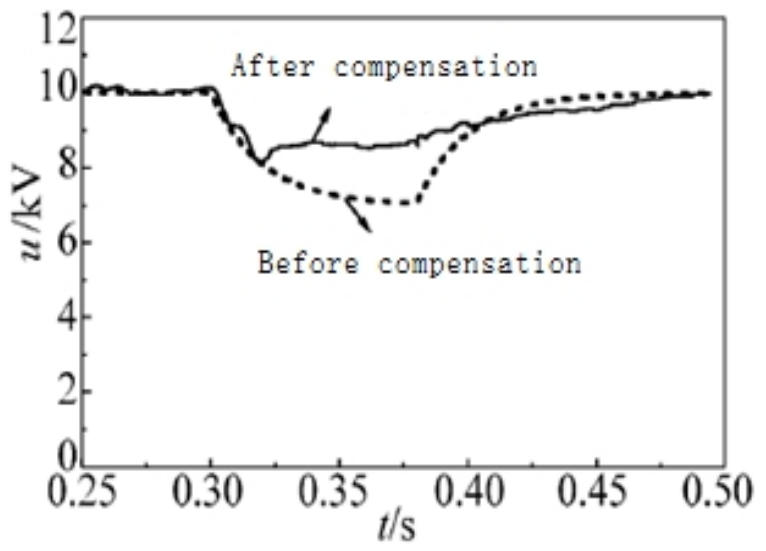

(b) Voltage sag compensation

Fig. 5 Compensation of LVRT

\section{Conclusions}

Coordinated power control method for HB-PCS based battery storage is put forward in this paper, and simulation models for 10MW PV station and $1 \mathrm{MW}$ storage system joint operation is built, meanwhile, a variety of simulation cases are studied.

Simulation results show that coordinated power control method can make full use of control ability of active and reactive power four quadrants for storage system, and stabilize power output fluctuation from PV station to meet injection requirements. Coordinated power control method also may make voltage at grid-connected point stable and improve power quality. It is helpful to promote low voltage ride through capability as well. If it runs in STATCOM mode, it can offer reactive power support to the grid to some extent, which is equivalent of a dual-purpose machine to save investment.

\section{References}

[1] Wang Kai,Li Wenyi and Zhang Xiaofei, in: Power System Technology, Vol. 37 ( 2013), p.3096

[2] Wang Haining, Su Jianhui and Ding Ming, in: Proceedings of the CSEE, Vol. 27(2007), p.75

[3] Yoshimoto K, NanaharaT and Koshimizu G, New control method for regulating state-of-charge of a battery in hybrid wind power /battery energy storage system//PSCE, Tokyo, Japan, p.1224(2006)

[4] Zhang Weidong and Shen Lan, In: Electric Power Automation Equipment, Vol. 33 (2013), p. 106

[5] Bao Xuena and Xu ming, in: Automation of Electric Power Systems, Vol. 37 (2013), p. 115

[6] Wang Haini, in: Transactions of China Electrotechnical Society, Vol. 20(2015), p.114

[7] Kong Lingguo and Yang Deyou, in: Power System Technology, Vol. 37(2013), p. 312

[8] Chang Qian, in: IEEE Power Engineering Society Winter Meeting, New York, America(2002)

[9] Hill C A, Such M C and Chen D, in: IEEE Transactions on Smart Grid, Vol. 3 (2012), p. 850

[10] Miao Qing and Ai Hongke, In: Asia-Pacific Conference on Electrical Engineering and Automation(2013)

[11] Wu Junyong, Ai Hongke and Hao Liangliang, China Invention Patent . 201,310,489,329. ( 2013)

[12] Zhang Bingbing, and Liu Nian, in: Power System Protection and Control, Vol.41 (2013), p.103

[13] Zhang Ye and Jia Hongjie, in: Automation of Electric Power Systems, Vol. 36(2012), p. 36 